\title{
Methanol and water masers in IRAS 20126+4104: the distance, the disk, and the jet ${ }^{\star}$
}

\author{
L. Moscadelli ${ }^{1}$, R. Cesaroni ${ }^{1}$, M. J. Rioja ${ }^{2,3}$, R. Dodson $^{2}$, and M. J. Reid ${ }^{4}$ \\ 1 Osservatorio Astrofisico di Arcetri, INAF, Largo E. Fermi 5, 50125 Firenze, Italy \\ e-mail: [cesa;mosca]@arcetri.astro.it \\ 2 International Centre for Radio Astronomy Research, University of Western Australia, Perth, Australia \\ 3 Observatorio Astronómico Nacional, Apdo. 112, 28803 Alcalá de Henares, Madrid, Spain \\ ${ }^{4}$ Harvard-Smithsonian Center for Astrophysics, 60 Garden Street, Cambridge, MA 02138, USA
}

Received 26 August 2010 / Accepted 19 November 2010

ABSTRACT

Context. Knowledge of the distance to high-mass star forming regions is crucial to obtain accurate luminosity and mass estimates of young OB-type (proto)stars and thus better constrain their nature and age. IRAS 20126+4104 is a special case, being the best candidate of a high-mass (proto)star surrounded by an accretion disk. Such a fact may be used to set constraints on theories of highmass star formation, but requires confirmation that the mass and luminosity of IRAS 20126+4104 are indeed typical of a B0.5 star, which in turn requires an accurate estimate of the distance.

Aims. The goal of our study is twofold: to determine the distance to IRAS 20126+4104, using the parallax of $\mathrm{H}_{2} \mathrm{O}$ masers associated

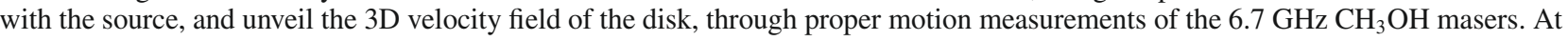
the same time, we can also obtain an estimate of the systemic velocity in the plane of the sky of the disk+star system.

Methods. We used the Very Long Baseline Array and the European VLBI Network to observe the $22.2 \mathrm{GHz} \mathrm{H}_{2} \mathrm{O}$ and $6.7 \mathrm{GHz}$ $\mathrm{CH}_{3} \mathrm{OH}$ masers in IRAS 20126+4104 at a number of epochs suitably distributed in time. The absolute positions of the maser features were established with respect to reference quasars, which allowed us to derive absolute proper motions.

Results. From the parallax of the $\mathrm{H}_{2} \mathrm{O}$ masers we obtain a distance of $1.64 \pm 0.05 \mathrm{kpc}$, which is very similar to the value adopted so far in the literature $(1.7 \mathrm{kpc})$ and confirms that IRAS $20126+4104$ is a high-mass (proto)star. From the $\mathrm{CH}_{3} \mathrm{OH}$ masers we derive the component in the plane of the sky of the systemic velocity of the disk + star system $\left(-16 \mathrm{~km} \mathrm{~s}^{-1}\right.$ in right-ascension and $+7.6 \mathrm{~km} \mathrm{~s}$ in declination). Accurate knowledge of the distance and systemic velocity allows us to improve on the model fit to the $\mathrm{H}_{2} \mathrm{O}$ maser jet presented in a previous study. Finally, we identify two groups of $\mathrm{CH}_{3} \mathrm{OH}$ maser features, one undergoing rotation in the disk and possibly distributed along a narrow ring centered on the star, the other characterised by relative proper motions indicating that the features are moving away from the disk, perpendicular to it. We speculate that the latter group might be tracing the disk material marginally entrained by the jet.

Conclusions. VLBI multi-epoch observations with phase referencing are confirmed to be an excellent tool for distance determinations and for the investigation of the structure and 3D velocity field within a few $100 \mathrm{AU}$ from newly born high-mass stars.

Key words. stars: formation - ISM: individual objects: IRAS 20126+4104 - masers - proper motions

\section{Introduction}

The role of disks in the formation of high-mass (i.e. O- and early B-type) stars has been extensively discussed in a number of reviews (see e.g. Cesaroni et al. 2006, 2007). Disks are important because they focus the accretion onto the forming star and allow some of the stellar photons to escape along the disk axis (see Krumholz et al. 2009, and references therein). The combined effects of these two mechanisms might prevent radiation pressure from halting the infalling gas, thus allowing growth of the stellar mass beyond the otherwise maximum value of $\sim 8 M_{\odot}$ (Palla \& Stahler 1993). For these reasons, the detection of disks around early-type (proto)stars could be used to prove that the formation of such stars is a scaled up version of that of solar-type stars.

Despite the numerous searches made in recent years with (sub)millimeter interferometers, no true (i.e. self-gravitating, dynamically stable) circumstellar disk has been found associated with early O-type (proto)stars. There is, however, growing evidence of Keplerian disks around early B-type stars and

* Based on observations carried out with the NRAO Very Large Baseline Array and European VLBI Network.
IRAS $20126+4104$ is an exemplary case. This $10^{4} L_{\odot}$ young stellar object (YSO) has been the subject of a long series of studies, with angular resolution spanning from a few $10^{\prime \prime}$ to a few milli$\operatorname{arcsec}$ (mas). Here, we note some findings most relevant to the present article, namely:

- IRAS 20126+4104 is associated with a Keplerian disk rotating about a $\sim 7 M_{\odot}$ protostar (Cesaroni et al. 1997, 1999, 2005; Zhang et al. 1998);

- the disk is also seen in silhouette as a dark lane at nearand mid-IR wavelengths (Sridharan et al. 2005; De Buizer et al. 2007);

- the YSO is powering a bipolar jet/outflow, imaged in a variety of tracers from $\sim 1^{\prime}$ down to $\sim 100$ mas from the star (Wilking et al. 1990; Cesaroni et al. 1997, 1999, 2005; Shepherd et al. 2000; Su et al. 2007; Shinnaga et al. 2008; Hofner et al. 1999, 2007; Moscadelli et al. 2000, 2005; Trinidad et al. 2005);

- the jet/outflow appears to be undergoing precession (Shepherd et al. 2000; Cesaroni et al. 2005; Lebrón et al. 2006), perhaps caused by the presence of another YSO 
detected at radio and IR wavelengths (Hofner et al. 1999; Sridharan et al. 2005).

Such an overwhelming quantity of information made it possible to determine a number of physical parameters of the star-diskoutflow system. Among these, the most important are the stellar mass and luminosity, which allows one to identify this object as an early B-type protostar. However, the values on which this conclusion is based depend on the distance, $d$, which is poorly known. The value quoted in the literature $(1.7 \mathrm{kpc})$ was adopted under the assumption that IRAS 20126+4104 lies in the Cyg-X region (see Shinnaga et al. 2008, for a discussion of the distance determination). In a previous study, we tried to constrain the distance to the source using the observed proper motions of the $\mathrm{H}_{2} \mathrm{O}$ maser spots associated with it (see Moscadelli et al. 2005, for details). We concluded that IRAS 20126+4104 should be located farther than $1.2 \mathrm{kpc}$, but this result, beside being a very loose constraint, is also model dependent. In conclusion, one cannot exclude the possibility that this YSO is much less massive than expected, if $d \ll 1.7 \mathrm{kpc}$, or much more, if $d \gg 1.7 \mathrm{kpc}$. The former possibility would dramatically reduce the values of stellar mass and luminosity, thus "destroying" the most convincing example of an accretion disk around an early B-type protostar. In turn, this would have implications for the formation scenario of high-mass stars, as previously explained. Hence, it is of fundamental importance to obtain an accurate estimate of $d$. Recently, several studies (see Reid et al. 2009b, and references therein) have proved the potential of maser parallax measurements for distance determinations. We have applied this method to the $\mathrm{H}_{2} \mathrm{O}$ masers in IRAS 20126+4104 and in Sect. 4 we report on the results obtained.

Another crucial issue in Galactic studies is that of the reference system used to determine absolute proper motions. In the case of IRAS 20126+4104, it is necessary to refer the measurement to the star. Usually, for this purpose the absolute proper motion is measured by referring the spot position to a background quasar. Then the apparent motions due to the annual parallax and motion of the source with respect to the Sun are subtracted. The result, however, is not very precise, as the corrections depend on the distance to the source and the accuracy of the Solar motion and Galactic rotation curve adopted. In the case of IRAS 20126+4104, the distance can be determined from the parallax measurement of the $\mathrm{H}_{2} \mathrm{O}$ maser spots (as shown in the present paper) and the major source of uncertainty remains the reliability of the Galactic rotation curve, for which deviations of $\sim 15 \mathrm{~km} \mathrm{~s}^{-1}$ are known to exist (see Reid et al. 2009b). This problem would be solved if one could directly measure the proper motion of the star and then subtract this from that of the maser spots. In Sect. 5 we will show that the $\mathrm{CH}_{3} \mathrm{OH}$ masers in IRAS $20126+4104$ can be used for this purpose.

Finally, in Sect. 6 knowledge of the distance and 3D velocities of the $\mathrm{H}_{2} \mathrm{O}$ and $\mathrm{CH}_{3} \mathrm{OH}$ maser spots will be used to analyse the detailed structure of the disk-jet system in IRAS $20126+4104$ on scales as small as $\sim 150$ AU from the protostar.

\section{Observations and calibration}

We conducted multi-epoch, VLBI observations of the $\mathrm{H}_{2} \mathrm{O}$ and $\mathrm{CH}_{3} \mathrm{OH}$ masers toward IRAS 20126+4104 at 22.2 and $6.7 \mathrm{GHz}$, respectively. To determine the maser absolute positions and velocities, we performed phase-referencing observations by fast switching between the maser source and the calibrator $\mathbf{J} 2007+4029$. This calibrator has an angular offset from the maser source of 1.5 and belongs to the list of sources defining the International Celestial Reference Frame (ICRF). Its absolute position is known to better than \pm 1 mas and its flux measured with the $\mathrm{NRAO}^{1}$ Very Long Baseline Array (VLBA) at $\mathrm{S}$ and $\mathrm{X}$ bands is 0.3 and $0.8 \mathrm{Jy}$ beam $^{-1}$, respectively. At each VLBI epoch, $\mathrm{H}_{2} \mathrm{O}$ and $\mathrm{CH}_{3} \mathrm{OH}$ maser absolute positions have been derived by imaging the maser visibilities after applying the phase and amplitude corrections derived working with the strong calibrator J2007+4029. The estimated accuracy of the maser position offsets is $0.05-0.1$ mas and $\sim 0.3$ mas for the $\mathrm{H}_{2} \mathrm{O}$ and $\mathrm{CH}_{3} \mathrm{OH}$ masers, respectively, the better accuracy of the water maser positions owing to the improved calibration of the delays introduced by the Earth's atmosphere (see Sect. 2.1).

Six fringe finders (J0237+2848; 3C 345; J1800+3848; $\mathrm{J} 2002+4725 ; \quad \mathrm{J} 2005+7752 ; \quad 3 \mathrm{C} 454.3)$ were observed for bandpass, single-band delay, and instrumental phase-offset calibration. Data were reduced with AIPS following the VLBI spectral line procedures. For a description of the general data calibration procedures and the criteria used to identify individual masing clouds, derive their parameters (position, intensity, flux and size), and measure their (relative and absolute) proper motions, we refer to the recent paper on VLBI observations of $\mathrm{H}_{2} \mathrm{O}$ and $\mathrm{CH}_{3} \mathrm{OH}$ masers by Sanna et al. (2010). For a description of the general observational setup and method of calibration of maser parallax observations, we refer to Reid et al. (2009a). The derived absolute proper motions have been corrected for the apparent proper motion due to the Earth's orbit around the Sun (parallax), the Solar Motion and the differential Galactic Rotation between our LSR and that of the maser source. We have adopted a flat Galaxy rotation curve, with a standard Solar motion of $19.5 \mathrm{~km} \mathrm{~s}^{-1}$ towards the Solar Apex at $\alpha(\mathbf{J} 2000)=271^{\circ}$ and $\delta(\mathbf{J} 2000)=29^{\circ}$, corresponding to $U, V, W=-10.4,14.8,7.3 \mathrm{~km} \mathrm{~s}^{-1}$ (see Mihalas \& Binney 1981), and the values of the Galactic Constants $\left(R_{0}=8.4 \mathrm{kpc}, \Theta_{0}=\right.$ $254 \mathrm{~km} \mathrm{~s}^{-1}$ ) as recently determined by Reid et al. (2009b). In the following we only report on the main observational parameters of our maser VLBI observations.

\section{1. $22.2 \mathrm{GHz} \mathrm{H}_{2} \mathrm{O}$ masers: VLBA parallax observations}

We observed IRAS 20126+4104 (tracking center: $\alpha(\mathrm{J} 2000)=20^{\mathrm{h}} 14^{\mathrm{m}} 26^{\mathrm{s}} .0253$ and $\left.\delta(\mathrm{J} 2000)=41^{\circ} 13^{\prime} 32^{\prime \prime} .666\right)$ with the VLBA in the $6_{16}-5_{23} \mathrm{H}_{2} \mathrm{O}$ transition (rest frequency $22.23507985 \mathrm{GHz}$ ). The observations (program code: BM269) consisted of 5 epochs: January 28, May 24, August 2, November 5, 2008, and January 26, 2009. These dates were selected to symmetrically sample both the eastward and northward parallax signatures and minimize correlations among the parallax and proper motion parameters. At each epoch, in two $4 \mathrm{~h}$ blocks of phase-referencing observations (using a switching cycle between calibrators of $1 \mathrm{~min}$ ), we recorded both circular polarizations using two $8 \mathrm{MHz}$ bandwidths, with the higher-frequency bandwidth centered on a LSR velocity of $-3.0 \mathrm{~km} \mathrm{~s}^{-1}$. Geodetic-style observing blocks of $40 \mathrm{~min}$ were placed before the start, in the middle, and after the end of our phase-reference observations, in order to monitor slow changes in the total atmospheric delay for each telescope. The data were processed with the VLBA FX correlator in two correlation passes using either 512 or 16 spectral channels for the maser

\footnotetext{
1 The National Radio Astronomy Observatory is operated by Associated Universities, Inc., under cooperative agreement with the National Science Foundation.
} 
line and calibrator data, respectively. In each correlator pass, the data averaging time was $1 \mathrm{~s}$.

The natural CLEAN beam was an elliptical Gaussian with a $F W H M$ size of about 0.7 mas $\times 0.4$ mas at a PA of about $-15^{\circ}$ (east of north), slightly varying from epoch to epoch. To better compare maser images across the epochs, maser maps have been reconstructed using a round beam with a $F W H M$ size of 1 mas. Using an on-source integration time of about $4 \mathrm{~h}$, the rms noise level on the channel maps (evaluated over an area where no signal is present) was $\sim 5 \mathrm{mJy}^{\text {beam }}{ }^{-1}$. With 512 correlator channels, the $8 \mathrm{MHz}$ band provides a resolution in the line-of-sight velocity of $0.2 \mathrm{~km} \mathrm{~s}^{-1}$.

\section{2. $22.2 \mathrm{GHz} \mathrm{H}_{2} \mathrm{O}$ masers: single-epoch EVN observation}

We observed IRAS $20126+4104$ (tracking center: $\alpha($ J2000) $=$ $20^{\mathrm{h}} 14^{\mathrm{m}} 26^{\mathrm{s}} 0387$ and $\left.\delta(\mathrm{J} 2000)=41^{\circ} 13^{\prime} 32^{\prime \prime} .534\right)$ with the European VLBI Network ${ }^{2}(\mathrm{EVN})$ in the $\mathrm{H}_{2} \mathrm{O} 22.2 \mathrm{GHz}$ maser line on March 42009 (program code: EM059D). The antennas involved in the observations were Jodrell2, Effelsberg, Medicina, Noto, Metsahovi, Onsala, Yebes and Shanghai. During a total run of $13 \mathrm{~h}$ (interspersing phase-referencing, maser-calibrator observations every $1.5 \mathrm{~h}$ with scans on the fringe-finders), we recorded both circular polarizations using a bandwidth of $16 \mathrm{MHz}$, centered on a LSR velocity of $-3.5 \mathrm{~km} \mathrm{~s}^{-1}$. The data were processed with the MKIV correlator at the Joint Institute for VLBI in Europe (JIVE - Dwingeloo, The Netherlands) using an averaging time of $2 \mathrm{~s}$ and 1024 spectral channels for each observing bandwidth.

The natural CLEAN beam was an elliptical Gaussian with a $F W H M$ size of 1.5 mas $\times 1.1$ mas at a PA of $83^{\circ}$. With an onsource integration time of about $4 \mathrm{~h}$, the rms noise level on the channel maps was about $30 \mathrm{mJy}^{\text {beam }}{ }^{-1}$. With 1024 correlator channels, the $16 \mathrm{MHz}$ band provides a resolution in the line-ofsight velocity of $0.2 \mathrm{~km} \mathrm{~s}^{-1}$.

\section{3. $6.7 \mathrm{GHz} \mathrm{CH}_{3} \mathrm{OH}$ masers: multi-epoch EVN observations}

We observed IRAS $20126+4104$ (tracking center: $\alpha($ J2000) $=$ $20^{\mathrm{h}} 14^{\mathrm{m}} 26^{\mathrm{s}} .05$ and $\left.\delta(\mathrm{J} 2000)=41^{\circ} 13^{\prime} 32^{\prime \prime} \cdot 7\right)$ with the European VLBI Network (EVN) in the $51_{1}-6_{0} \mathrm{~A}^{+} \mathrm{CH}_{3} \mathrm{OH}$ transition (rest frequency $6.66851920 \mathrm{GHz}$ ), at two epochs (program codes: EM064C and EM064D), separated by about $2 \mathrm{yr}$, observed on March 21, 2007 and March 11, 2009. At both epochs, antennas involved in the observations were Cambridge, Jodrell2, Effelsberg, Onsala, Medicina, Noto, Torun and Westerbork. During a run of about $6 \mathrm{~h}$ per epoch (alternating phasereferencing, maser-calibrator observations every $1.5 \mathrm{~h}$ with scans on the fringe-finders), we recorded both circular polarizations using eight $2 \mathrm{MHz}$ bandwidths, with the third (frequencyordered) band centered on the maser target LSR velocity of $-6.1 \mathrm{~km} \mathrm{~s}^{-1}$. The total $16 \mathrm{MHz}$ bandwidth for each polarization was effective to increase the SNR on the continuum calibrator. The data were processed with the MKIV correlator at the Joint Institute for VLBI in Europe (JIVE - Dwingeloo, The Netherlands) in two correlation passes using either 1024 or 128 spectral channels for the maser line and calibrator data, respectively. In each correlator pass, the data averaging time was $1 \mathrm{~s}$.

The natural CLEAN beam was an elliptical Gaussian with a $F W H M$ size of about 8 mas $\times 6$ mas at a PA of about $\sim 45^{\circ}$,

\footnotetext{
2 The European VLBI Network is a joint facility of European, Chinese and other radio astronomy institutes founded by their national research councils.
}

slightly varying between the two epochs. Using an on-source integration time of about $2.2 \mathrm{~h}$, the rms noise level on the channel maps was about 6 mJy beam ${ }^{-1}$. With 1024 correlator channels, the $2 \mathrm{MHz}$ band provides a resolution in the line-of-sight velocity of $0.09 \mathrm{~km} \mathrm{~s}^{-1}$.

In order to unambiguously determine maser spot persistency and derive reliable proper motions, it is fundamental to observe (at least) three different epochs (see discussion in Sanna et al. 2010). For this purpose, we have extracted from the EVN archive and analysed phase-referencing observations of the 6.7 $\mathrm{GHz} \mathrm{CH}_{3} \mathrm{OH}$ masers towards IRAS 20126+4104 observed on November 62004 (program code: EL032). The main parameters of these observations closely match those of our two EVN epochs. The selected calibrator, J2007+4029, is the same intense quasar. The EVN antennas used are the same as in the programs EM064C and EM064D, with the exception that the Darnhall telescope was used instead of Jodrell2. For the maser emission, the observing bandwidth, $2 \mathrm{MHz}$, and the correlator spectral channels, 1024, are the same as employed in our, more recent, EVN experiments. As a result, the angular and line-ofsight velocity resolution, and the sensitivity of the EL032 data are comparable to those of the EM064C and EM064D experiments, and the three EVN observations constitute a suitable dataset to study the maser motions.

\section{Results}

While many $\mathrm{H}_{2} \mathrm{O}$ maser features have been detected at the different epochs, only two of them persisted long enough (over 5 and 6 epochs) to be used for our parallax measurements. Details of the method adopted to derive a distance estimate will be given in Sect. 4. Since in the present article our interest is focused on $\mathrm{H}_{2} \mathrm{O}$ masers as tools to determine the distance to IRAS 20126+4104, we will not report on the results obtained for the other shorterlived water maser features.

As for methanol masers, Table 1 gives the main properties of the detected $\mathrm{CH}_{3} \mathrm{OH}$ maser features. In particular, Col. 1 gives the feature identification number, Col. 2 the observing epochs at which the feature was detected, Cols. 3 and 4 the position offsets with the associated errors along the RA and Dec axes, relative to the reference feature 3, Col. 4 the flux density, Col. 5 the LSR velocity, Cols. 6 and 7 the relative proper motion components with the associated errors along the RA and Dec axes, measured with respect to the reference feature 3 , and Cols. 8 and 9 the absolute proper motion components with the associated errors along the RA and Dec axes. Note that the positions given in Cols. 3 and 4 are measured at the first of the epochs reported in Col. 2, while the quantities given in Cols. 5 and 6 are the mean values over all the observing epochs. The absolute positions of feature 3 at the three observing epochs are given in the caption of Table 1 . We stress that proper motions evaluated using only two epochs are affected by much larger uncertainties (with errors from 0.6 up to $3 \mathrm{~km} \mathrm{~s}^{-1}$ ), than proper motions calculated using three epochs (typical errors are $0.3 \mathrm{~km} \mathrm{~s}^{-1}$ ). The uncertainty is mainly due to random variations in the spatial and velocity structure of the maser emission. One needs at least three epochs to average out this effect. Therefore, all the reported methanol maser proper motions have been calculated using three epochs.

For the sake of clarity, it is important to point out what is meant with "relative" and "absolute" in the present study. When used for positions, the term relative refers to measurements with respect to a maser feature, while absolute indicates measurements with respect to an external reference (the background quasar), whose position is known to a high degree of accuracy. 
Table 1. Parameters of the $6.7 \mathrm{GHz} \mathrm{CH}_{3} \mathrm{OH}$ maser features in IRAS $20126+4104$.

\begin{tabular}{|c|c|c|c|c|c|c|c|c|c|}
\hline $\begin{array}{l}\text { Feature } \\
\text { number }\end{array}$ & $\begin{array}{c}\text { Epochs of } \\
\text { detection }\end{array}$ & $\begin{array}{c}\Delta \alpha \\
(\mathrm{mas})\end{array}$ & $\begin{array}{c}\Delta \delta \\
(\mathrm{mas})\end{array}$ & $\begin{array}{l}S_{v} \\
(\mathrm{Jy})\end{array}$ & $\begin{array}{c}V_{\mathrm{LSR}} \\
\left(\mathrm{km} \mathrm{s}^{-1}\right)\end{array}$ & $\begin{array}{c}v_{\alpha} \\
\left(\mathrm{km} \mathrm{s}^{-1}\right)\end{array}$ & $\begin{array}{c}v_{\delta} \\
\left(\mathrm{km} \mathrm{s}^{-1}\right)\end{array}$ & $\begin{array}{c}V_{\alpha} \\
\left(\mathrm{km} \mathrm{s}^{-1}\right)\end{array}$ & $\begin{array}{c}V_{\delta} \\
\left(\mathrm{km} \mathrm{s}^{-1}\right)\end{array}$ \\
\hline 1 & $1,2,3$ & $-233.00 \pm 0.08$ & $-5.79 \pm 0.08$ & 40.5 & -6.1 & $-2.9 \pm 0.2$ & $0.2 \pm 0.2$ & $-16.6 \pm 0.8$ & $6.4 \pm 0.8$ \\
\hline 2 & $1,2,3$ & $-40.54 \pm 0.09$ & $31.24 \pm 0.09$ & 5.8 & -7.7 & $-2.4 \pm 0.2$ & $0.9 \pm 0.2$ & $-16.1 \pm 0.8$ & $7.2 \pm 0.8$ \\
\hline 3 & $1,2,3$ & 0.00 & 0.00 & 4.9 & -7.1 & 0.0 & 0.0 & $-13.6 \pm 0.8$ & $6.2 \pm 0.8$ \\
\hline 4 & $1,2,3$ & $-171.13 \pm 0.10$ & $-19.35 \pm 0.10$ & 3.7 & -6.5 & $-4.8 \pm 0.2$ & $3.5 \pm 0.2$ & $-18.4 \pm 0.8$ & $9.6 \pm 0.8$ \\
\hline 5 & 1,3 & $-23.75 \pm 0.30$ & $27.79 \pm 0.29$ & 3.4 & -7.6 & - & - & - & - \\
\hline 6 & 3 & $-3.28 \pm 0.08$ & $-0.11 \pm 0.09$ & 2.8 & -6.8 & - & - & - & - \\
\hline 7 & 1,3 & $-31.45 \pm 0.30$ & $27.94 \pm 0.33$ & 2.6 & -7.6 & - & - & - & - \\
\hline 8 & $1,2,3$ & $-152.15 \pm 0.20$ & $-17.98 \pm 0.21$ & 2.5 & -6.7 & $-3.5 \pm 0.3$ & $1.5 \pm 0.3$ & $-15.7 \pm 0.8$ & $8.1 \pm 0.8$ \\
\hline 9 & $1,2,3$ & $-246.24 \pm 0.26$ & $-1.89 \pm 0.24$ & 2.5 & -6.8 & $-4.9 \pm 0.4$ & $1.8 \pm 0.4$ & $-16.7 \pm 0.9$ & $7.8 \pm 0.9$ \\
\hline 10 & $1,2,3$ & $43.46 \pm 0.10$ & $-1.48 \pm 0.10$ & 1.7 & -8.3 & $-1.3 \pm 0.2$ & $-0.4 \pm 0.2$ & $-14.9 \pm 0.8$ & $5.9 \pm 0.8$ \\
\hline 11 & $1,2,3$ & $-79.31 \pm 0.10$ & $-113.86 \pm 0.10$ & 1.1 & -4.9 & $-0.4 \pm 0.2$ & $5.4 \pm 0.2$ & $-14.1 \pm 0.8$ & $11.7 \pm 0.8$ \\
\hline 12 & 1,2 & $-16.71 \pm 0.16$ & $3.96 \pm 0.17$ & 1.0 & -6.9 & - & - & - & - \\
\hline 13 & 3 & $-11.51 \pm 0.25$ & $-34.93 \pm 0.16$ & 0.9 & -7.6 & - & - & - & - \\
\hline 14 & 2,3 & $-215.05 \pm 0.23$ & $-12.07 \pm 0.19$ & 0.9 & -6.3 & - & - & - & - \\
\hline 15 & 2,3 & $-30.22 \pm 0.11$ & $2.63 \pm 0.11$ & 0.9 & -7.1 & - & - & - & - \\
\hline 16 & $1,2,3$ & $-176.95 \pm 0.17$ & $-29.11 \pm 0.17$ & 0.9 & -5.6 & $-2.8 \pm 0.3$ & $1.3 \pm 0.3$ & $-16.0 \pm 0.8$ & $7.9 \pm 0.8$ \\
\hline 17 & $1,2,3$ & $-180.45 \pm 0.11$ & $-52.37 \pm 0.12$ & 0.8 & -5.1 & $-2.9 \pm 0.3$ & $-1.1 \pm 0.3$ & $-16.4 \pm 0.8$ & $5.1 \pm 0.8$ \\
\hline 18 & 3 & $-77.79 \pm 0.15$ & $6.46 \pm 0.14$ & 0.7 & -6.7 & - & - & - & - \\
\hline 19 & 3 & $-150.64 \pm 0.25$ & $-80.58 \pm 0.21$ & 0.6 & -6.6 & - & - & - & - \\
\hline 20 & 1,2 & $27.36 \pm 0.23$ & $-0.98 \pm 0.23$ & 0.5 & -7.8 & - & - & - & - \\
\hline 21 & 3 & $-140.40 \pm 0.15$ & $-12.30 \pm 0.12$ & 0.4 & -5.5 & - & - & - & - \\
\hline 22 & 3 & $-214.16 \pm 0.21$ & $-5.22 \pm 0.17$ & 0.4 & -6.6 & - & - & - & - \\
\hline 23 & 3 & $-152.48 \pm 0.13$ & $-11.28 \pm 0.14$ & 0.4 & -5.6 & - & - & - & - \\
\hline 24 & 1,2 & $7.47 \pm 0.38$ & $-3.15 \pm 0.40$ & 0.3 & -6.8 & - & - & - & - \\
\hline 25 & 2,3 & $-189.83 \pm 0.44$ & $-22.76 \pm 0.28$ & 0.2 & -5.8 & - & - & - & - \\
\hline 26 & 2,3 & $-32.69 \pm 0.85$ & $-75.49 \pm 0.65$ & 0.2 & -5.1 & - & - & - & - \\
\hline 27 & 3 & $46.71 \pm 0.15$ & $29.06 \pm 0.20$ & 0.2 & -8.7 & - & - & - & - \\
\hline 28 & 3 & $36.86 \pm 0.14$ & $4.74 \pm 0.15$ & 0.2 & -8.3 & - & - & - & - \\
\hline 29 & 2,3 & $-212.65 \pm 0.36$ & $-16.60 \pm 0.36$ & 0.1 & -5.7 & - & - & - & - \\
\hline 30 & 3 & $-191.04 \pm 0.20$ & $-33.16 \pm 0.22$ & 0.1 & -5.2 & - & - & - & - \\
\hline 31 & 3 & $-228.56 \pm 0.38$ & $-19.26 \pm 0.40$ & 0.1 & -4.7 & - & - & - & - \\
\hline 32 & 2,3 & $-67.77 \pm 0.76$ & $-84.08 \pm 0.82$ & 0.1 & -5.2 & - & - & - & - \\
\hline 33 & 3 & $-44.31 \pm 0.51$ & $-72.72 \pm 0.40$ & 0.1 & -4.6 & - & - & - & - \\
\hline
\end{tabular}

Notes. The offsets, $\Delta \alpha$ and $\Delta \delta$, of a given feature are measured at the epoch (given in Col. 2) when the feature was detected for the first time, and are computed with respect to the position of feature 3 at the same epoch. The positions of feature 3 at the three observing epochs are: $\alpha(\mathrm{J} 2000)=20^{\mathrm{h}} 14^{\mathrm{m}} 26^{\mathrm{s}} .07111, \delta(\mathrm{J} 2000)=41^{\circ} 13^{\prime} 32^{\prime \prime} \cdot 701$, at epoch $1($ November 6,2004$) ; \alpha(\mathrm{J} 2000)=20^{\mathrm{h}} 14^{\mathrm{m}} 26^{\mathrm{s}} .07026, \delta(\mathrm{J} 2000)=41^{\circ} 13^{\prime} 32^{\prime \prime} \cdot 691$, at epoch $2\left(\right.$ March 21, 2007); $\alpha(\mathrm{J} 2000)=20^{\mathrm{h}} 14^{\mathrm{m}} 26^{\mathrm{s}} .06963, \delta(\mathrm{J} 2000)=41^{\circ} 13^{\prime} 32^{\prime \prime}$ 683, at epoch 3 (March 11, 2009). The errors on these positions are in all cases $<1$ mas. The relative proper motions, $v_{\alpha}$ and $v_{\delta}$ also refer to feature 3 .

In the case of proper motions, relative means that the proper motion is measured using a maser feature as a reference, whereas absolute indicates proper motions obtained by referring the motion to the background quasar, and then subtracting the contribution of the parallax, Solar motion with respect to the LSR, and galactic rotation. This is made in an attempt to obtain the proper motion, and thus the velocity, relative to the protostar. However, the absolute proper motions thus obtained require an additional correction, for which we refer to the discussion in Sect. 5 .

\section{The distance to IRAS 20126+4104}

Targets of our parallax measurements are compact water maser spots persistent across the six VLBI (five VLBA and one EVN) epochs. Looking at the maser spectra of each epoch, persistent emission could be identified only near the systemic velocity at $3.5 \mathrm{~km} \mathrm{~s}^{-1}$. Inspecting the images of each velocity channel, only two spots (at line-of-sight velocities of -6.4 and $-3.2 \mathrm{~km} \mathrm{~s}^{-1}$, respectively) maintaned a sufficiently compact structure to be used as astrometric targets. Such a low number of usable spots is not surprising, due to the well known variability of $\mathrm{H}_{2} \mathrm{O}$ maser emission (see e.g. Felli et al. 2007). Moreover, this emission happened to be especially faint in IRAS 20126+4104 during the period of our observations.

The results of the multi-epoch $\mathrm{H}_{2} \mathrm{O}$ measurements over $\sim 1.5 \mathrm{yr}$ are illustrated in Fig. 1. The top panel shows the positions of the two persistent maser features, one detected at all 6 epochs, the other only at 5 epochs. These have been fitted with a model taking into account the apparent motion due to the annual parallax plus a constant velocity vector: -10.3 mas $\mathrm{yr}^{-1}$ in

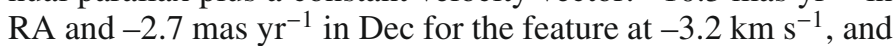

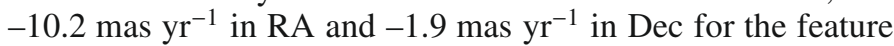
at $-6.4 \mathrm{~km} \mathrm{~s}^{-1}$. This vector accounts for both the motion of the local system (the star) relative to the Sun and the motion of the spots relative to the star. The assumption of constant velocity has proved successful in similar experiments (see Reid et al. 2009b, and references therein) and in the case of IRAS 20126+4104 is supported by the conical jet model of Moscadelli et al. (2000, 2005). The curves in Fig. 1 are the best fit to the data, corresponding to a parallax of $00^{\prime} 61 \pm 0.02$, i.e. a distance of $1.64 \pm 0.05 \mathrm{kpc}$. In the bottom panel of the figure, we show the residual position offsets at the six epochs, after subtracting the contribution of the constant velocity vector.

The fundamental conclusion that can be drawn from this result is that the distance adopted so far in the literature $(1.7 \mathrm{kpc})$ 

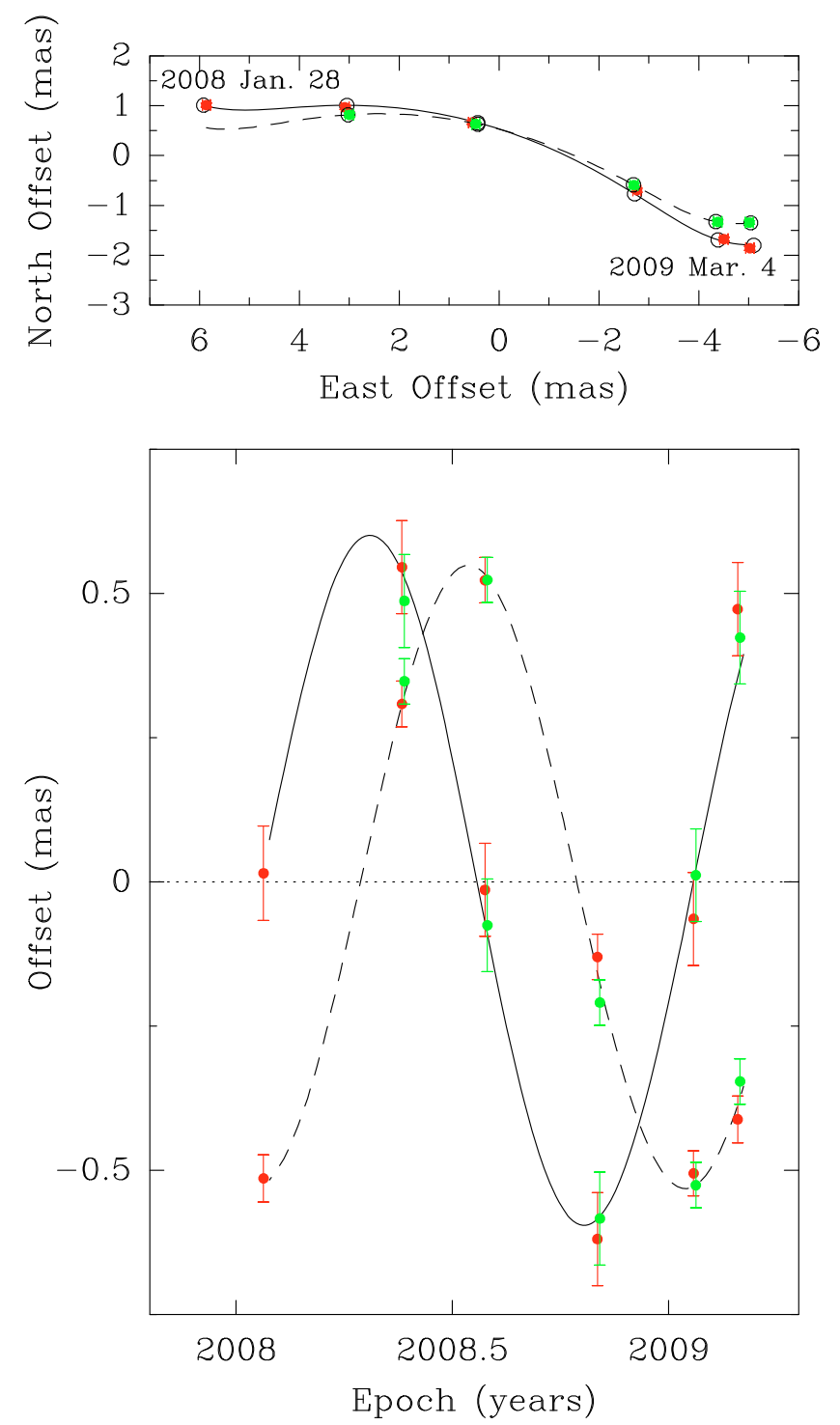

Fig. 1. Top. Positions of the two persistent $\mathrm{H}_{2} \mathrm{O}$ maser features in IRAS 20126+4104 at the six epochs of our VLBI observations. Note that the reported positions have been suitably offset from the measured positions for displaying purposes. Green and red circles indicate features at -6.4 and $-3.2 \mathrm{~km} \mathrm{~s}^{-1}$, respectively. Their positions at the $2^{\text {nd }}$ observing epoch are $\alpha(\mathrm{J} 2000)=20^{\mathrm{h}} 14^{\mathrm{m}} 26^{\mathrm{s}} .0165$ and $\delta(\mathrm{J} 2000)=41^{\circ} 13^{\prime} 32^{\prime \prime} .577$, for the feature at $-3.2 \mathrm{~km} \mathrm{~s}^{-1}$, and $\alpha(\mathrm{J} 2000)=20^{\mathrm{h}} 14^{\mathrm{m}} 26.0167$ and $\delta(\mathrm{J} 2000)=41^{\circ} 13^{\prime} 32^{\prime \prime} .576$, for the other. The solid and dashed curves with open circles are the best fits, consisting of the apparent motion due to the annual parallax for a common a distance of $1.64 \pm 0.05 \mathrm{kpc}$ for both features plus a constant velocity in the plane of the sky different for each feature (see text). Bottom. Residual motions in right ascension and declination, obtained after subtraction of the constant velocities. The curves are the fits consisting only of the annual parallax. The solid and dashed curves correspond, respectively, to the RA and Dec offsets.

for IRAS $20126+4104$ was correct. This confirms all the estimates of important physical parameters, in particular the stellar mass $\left(\sim 7 M_{\odot}\right)$ and bolometric luminosity $\left(\sim 10^{4} L_{\odot}\right)$. We can thus conclude that IRAS $20126+4104$ is indeed a B0.5 protostar surrounded by a Keplerian accretion disk (Cesaroni et al. 2005).

\section{The systemic velocity of IRAS $20126+4104$}

Methanol masers have been claimed by several authors to be associated with circumstellar disks (see e.g. Norris et al. 1998;
Minier et al. 2000; Pestalozzi et al. 2009). In IRAS 20126+4104 they appear to lie in the north-eastern part of the disk (Minier et al. 2001; Edris et al. 2005) and their velocities are blue-shifted, consistent with the Keplerian rotation pattern observed in thermal lines. Our EVN images are consistent with this finding, as illustrated in Fig. 2, where the $\mathrm{CH}_{3} \mathrm{OH}$ maser features are overlayed on the disk silouhette. The latter is very schematic and assumes a disk radius of $\sim 1000 \mathrm{AU}$ (Cesaroni et al. 2007) and the same center and inclination as the $\mathrm{H}_{2} \mathrm{O}$ maser jet (see Sect. 6.1).

If the masers lie in the disk and co-rotate with it, the corresponding velocity vectors should also lie in the plane of the disk. Since the latter is basically edge-on and the masers cluster is at a (projected) distance of $\sim 0.2$ or $340 \mathrm{AU}$ from the star, one would expect the proper motions to be relatively small ( $\$ 4 \mathrm{~km} \mathrm{~s}^{-1}$, assuming Keplerian rotation about a $7 M_{\odot}$ star) and directed parallel to the disk, i.e. NE-SW. In contrast, the absolute proper motions measured by us have speeds of $\sim 18 \mathrm{~km} \mathrm{~s}^{-1}$ and are all roughly perpendicular to the disk, as shown in Fig. 2. It is also worth noting that the dispersions of the $\mathrm{CH}_{3} \mathrm{OH}$ velocities both along the line of sight and in the plane of the sky are small compared to the mean speed of the features, unlike $\mathrm{H}_{2} \mathrm{O}$ masers whose velocities span an order of magnitude. Thus, we note that the $\mathrm{CH}_{3} \mathrm{OH}$ masers are moving much slower than the $\mathrm{H}_{2} \mathrm{O}$ masers.

Our explanation of all these facts is that the absolute proper motions of the $\mathrm{CH}_{3} \mathrm{OH}$ masers are dominated by a peculiar motion of about $10 \mathrm{~km} \mathrm{~s}^{-1}$ with respect to a circular Galactic orbit. Whether such a peculiar motion is due to a wrong estimate of the Solar motion with respect to the LSR, to an inadequate Galaxy rotation curve, or to a deviation from it, is irrelevant for our purposes. The important point is that the mean absolute proper motion of the $\mathrm{CH}_{3} \mathrm{OH}$ masers can be used to correct any absolute proper motion measurement and determine the true motion with respect to the disk+star system to within about $\pm 4 \mathrm{~km} \mathrm{~s}^{-1}$ (the expected rotational velocity). Incidentally, we note that deviations from the standard Galaxy rotation curve are quite common, as demonstrated by Reid et al. (2009b) in their study of the Galactic velocity field. Note also that the existence of this "spurious" velocity component of $\sim 18 \mathrm{~km} \mathrm{~s}^{-1}$ in the plane of the sky has less impact on $\mathrm{H}_{2} \mathrm{O}$ masers, due to the much larger velocities of the features (up to $\sim 100 \mathrm{~km} \mathrm{~s}^{-1}$ ).

In conclusion, our $\mathrm{CH}_{3} \mathrm{OH}$ measurements can be used (combined with the corresponding Doppler shifts) to determine the $3 \mathrm{D}$ velocity of the star+disk system. In practice, in order to obtain the velocity in the plane of the sky with respect to the star, any absolute proper motion must be corrected by subtracting the mean absolute proper motion of the $\mathrm{CH}_{3} \mathrm{OH}$ masers, i.e. $-16 \mathrm{~km} \mathrm{~s}^{-1}$ along the right-ascension axis and $+7.6 \mathrm{~km} \mathrm{~s}^{-1}$ along the declination.

\section{Disk and jet structure in IRAS 20126+4104}

Better knowledge of the distance and, especially, of the systemic 3D velocity of IRAS 20126+4104 allows us to improve on the model presented by Moscadelli et al. (2005). Also, one can accurately compare the distributions and velocities of the $\mathrm{CH}_{3} \mathrm{OH}$ masers with those of the $\mathrm{H}_{2} \mathrm{O}$ masers, and thus draw a better picture of the disk and jet structure and of their relationship.

Before proceeding to this comparison, it is worth noting that the two types of masers have been observed at very different epochs, namely November 9, 2000 for the $\mathrm{H}_{2} \mathrm{O}$ masers (Moscadelli et al. 2005) and November 6, 2004 for the $\mathrm{CH}_{3} \mathrm{OH}$ masers (this paper). The maser spots could have moved over 


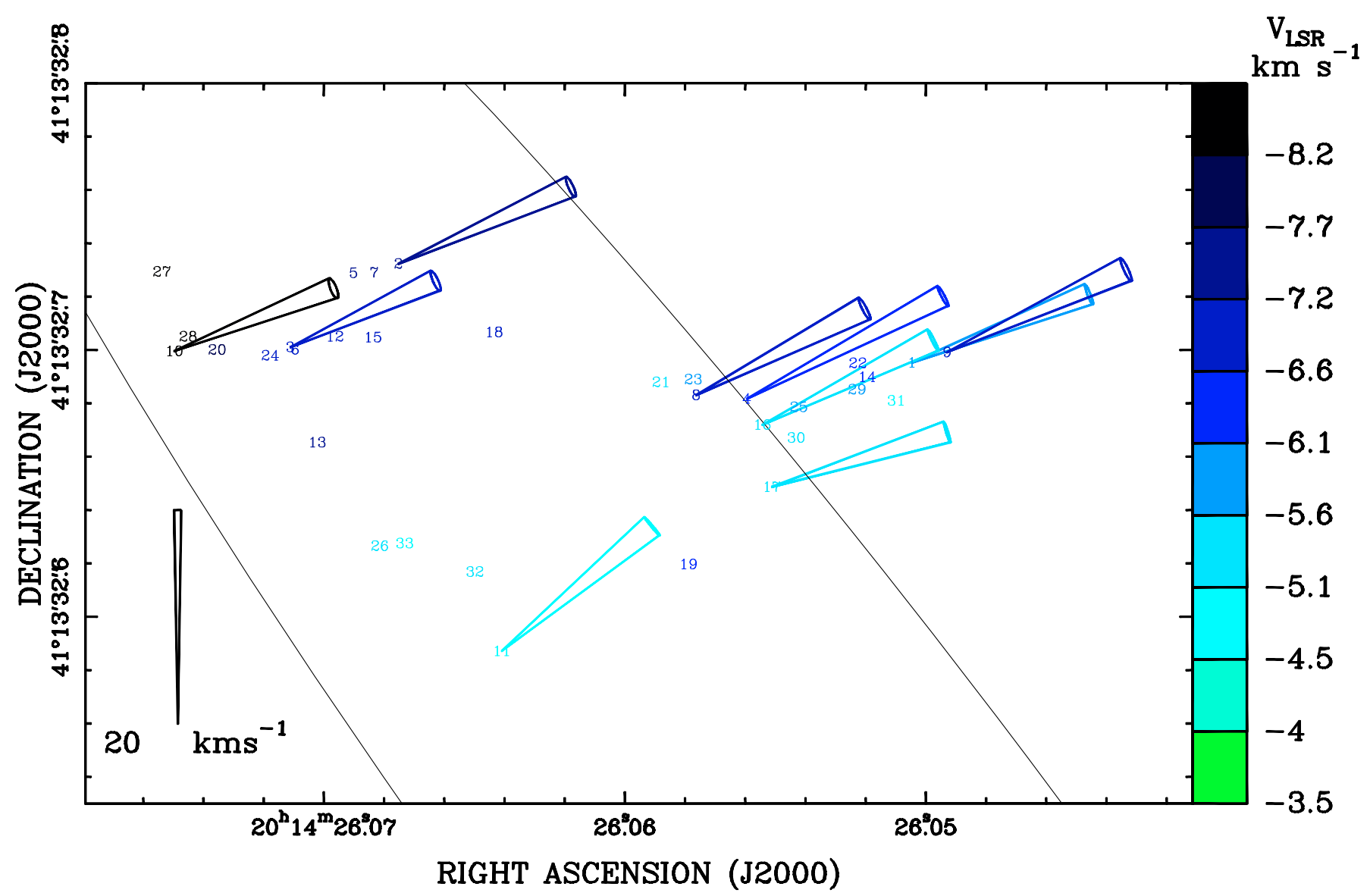

Fig. 2. Absolute proper motions (cones) of the $\mathrm{CH}_{3} \mathrm{OH}$ maser features (numbers) in IRAS 20126+4104. Numbers without an associated cone have been detected only over one or two epochs and the associated proper motion cannot be computed or is considered unreliable. Note that the all features are displayed at the positions of the first epoch (November 6, 2004). For features undetected at this epoch, the positions were obtained by extrapolation assuming they are moving with the mean absolute proper motion of the other features (in all cases the correction is $<10$ mas). Absolute proper motions have been corrected for parallax, motion of the Sun with respect to the LSR, and Galactic rotation adopting a flat rotation curve as explained in Sect. 2. Most of the mean residual proper motion $\left(\sim 18 \mathrm{~km} \mathrm{~s}^{-1}\right)$ is believed to be due to deviation of the source from the assumed rotation curve. The maser features are identified by the numbers given in Col. 1 of Table 1, while their colour indicates the LSR velocity according to the scale displayed to the right. The two arcs schematically outline the Keplerian disk (Cesaroni et al. 2005), whose center lies out of the figure, to the SW.

this $\sim 4$ yr period, thus questioning a positional comparison between the two maser types. However, from the known proper motions one can estimate, e.g., the expected mean position of the methanol masers at the observing epoch of the water masers, and we find that the displacement is only $\sim 8$ mas in RA and $\sim 18$ mas in Dec. Values that small are negligible with respect to the region of interest for us ( $\gtrsim 250$ mas, see Fig. 3).

\subsection{The conical $\mathrm{H}_{2} \mathrm{O}$ jet revised}

Moscadelli et al. (2005) interpreted the $\mathrm{H}_{2} \mathrm{O}$ masers as tracing the surface of a conical jet, expanding away from the star. The model could satisfactorily fit the observed line-of-sight velocities and absolute proper motions, after correcting for the parallax, motion of the Sun with respect to the LSR, and galactic rotation. Knowledge of the star's 3D velocity, obtained in Sect. 5, permits accurate estimation of the $\mathrm{H}_{2} \mathrm{O}$ maser velocities with respect to the star. Using this information, we could correct the proper motions of the $\mathrm{H}_{2} \mathrm{O}$ maser features derived by Moscadelli et al. (2005). The new velocity vectors are shown as arrows in Fig. 3. Comparison with Fig. 2 of Moscadelli et al. (2005) shows that the correction does not affect significantly the magnitudes and directions of the vectors, apart from the red-shifted feature to the SE. This had negligible proper motion in the old analysis of the data, whereas with the new correction it appears to move towards SE, lending further support to the bipolar jet model of the $\mathrm{H}_{2} \mathrm{O}$ masers. Note that the proper motions obtained in the present study for the two features selected for our parallax measurement in Sect. $4\left(-46 \mathrm{~km} \mathrm{~s}^{-1}\right.$ in RA and $11 \mathrm{~km} \mathrm{~s}^{-1}$ in Dec for the feature with LSR velocity of $-3.2 \mathrm{~km} \mathrm{~s}^{-1}$, and $47 \mathrm{~km} \mathrm{~s}^{-1}$ in RA and $17 \mathrm{~km} \mathrm{~s}^{-1}$ in Dec for that with LSR velocity of $-6.4 \mathrm{~km} \mathrm{~s}^{-1}$ ) are consistent with the jet-model proposed by Moscadelli et al. (2005). We have not derived the proper motions of the other features detected in the present experiment, because these will be the subject of a forthcoming paper illustrating the results of a VLBI multi-year monitoring of the water maser emission in IRAS 20126+4104.

We used the model of Moscadelli et al. (2005) to fit the new, corrected data and obtained new best-fit parameters that are qualitatively consistent with the old ones. We find a position angle (PA) of $115^{\circ}$ instead of $123^{\circ}$, an inclination with respect to the line of sight of $80^{\circ}$ instead of $96^{\circ}$, an opening angle of $9^{\circ}$ instead of $17^{\circ}$, and a velocity gradient along the jet of $0.116 \mathrm{~km} \mathrm{~s}^{-1} \mathrm{mas}^{-1}$ instead of $0.255 \mathrm{~km} \mathrm{~s}^{-1} \mathrm{mas}^{-1}$. Also, the position of the star is shifted by +143 mas in right ascension and -49 mas in declination. 


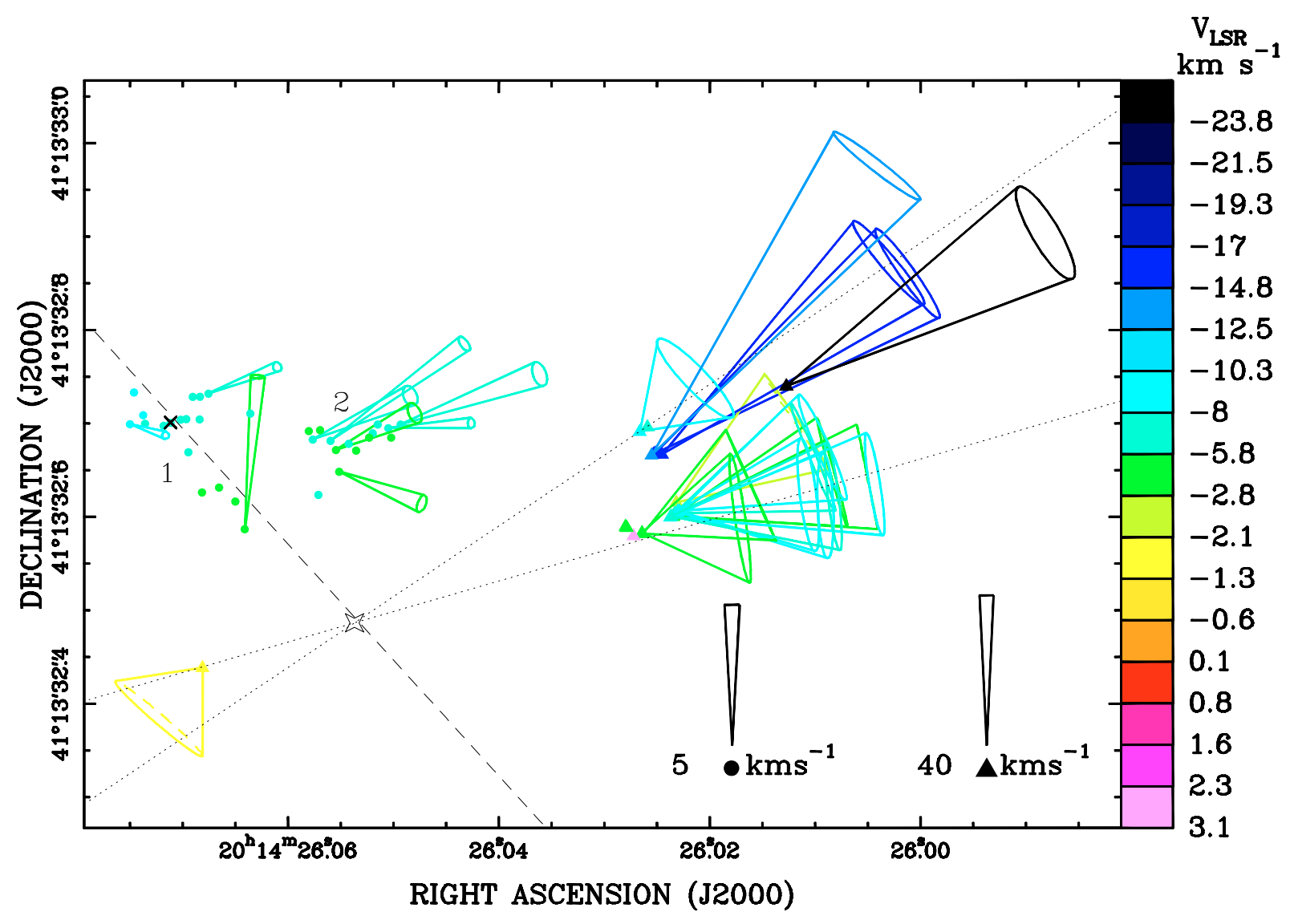

Fig. 3. Water (triangles) and $6.7 \mathrm{GHz}$ methanol (circles) masers in IRAS 20126+4104. The cones indicate the 3D velocities of the maser features with respect to the star, for the $\mathrm{H}_{2} \mathrm{O}$ masers, or relative to feature 3 (marked with a cross), for the $\mathrm{CH}_{3} \mathrm{OH}$ masers. Points without an associated cone have been detected only over one or two epochs and the associated proper motion cannot be computed or is considered unreliable. The cone opening angle corresponds to the $1 \sigma$ uncertainty, due to the error on the proper motions. The length of the cones is proportional to the speed, with different scales used for the two maser types as indicated in the figure. The colours correspond to the line of sight velocities, as shown in the colour scale to the right. The starred polygon marks the location of the star, obtained from the model fit to the $\mathrm{H}_{2} \mathrm{O}$ maser positions and 3D velocities. Labels " 1 " and " 2 " denote two elongated groups of $\mathrm{CH}_{3} \mathrm{OH}$ maser features. The dotted line outline the projection of the bipolar $\mathrm{H}_{2} \mathrm{O}$ maser jet onto the plane of the sky, while the dashed line is a linear fit to the $\mathrm{CH}_{3} \mathrm{OH}$ maser features of group 1 .

In conclusion, despite some differences between the old and new best-fit parameters, the corrected data confirm that the jet is very beamed, lies close to the plane of the sky, and is approximately perpendicular to the disk (whose PA is $\sim 53 \pm 7^{\circ}$, see Cesaroni et al. 2005).

\subsection{The disk-jet interface}

So far, we have used the $\mathrm{CH}_{3} \mathrm{OH}$ masers only to determine the systemic velocity of the star+disk system. Now, we analyse their role in tracing the distribution and kinematics of the gas in the disk-jet system.

Looking at Fig. 3, one sees that the $\mathrm{CH}_{3} \mathrm{OH}$ maser features are roughly outlining two linear structures, one oriented NESW and the other SE-NW; hereafter we will refer to these as, respectively, "group 1" and "group 2". The features belonging to these groups are (using the labels in Table 1): 2, 3, 5, 6, 7, 10, $11,12,13,15,18,20,24,26,27,28,32,33$ for group 1 and 1 , $4,8,9,14,16,17,19,21,22,23,25,29,30,31$ for group 2 . Comparison with Edris et al. (2005) lends support to this distinction, because both the spots positions and velocities appear consistent with those of our groups 1 and 2, with only one exception (spot number 15 in their Table 6).

We have calculated the relative proper motions (see Fig. 3) of the $\mathrm{CH}_{3} \mathrm{OH}$ maser features of both groups with respect to feature 3 , which belongs to group 1 and appears to be the most reliable for the persistency of the internal structure over the three observing epochs.

It is worth noting two facts. First, the masers in group 2 move towards NW, i.e. in the same direction as the blue lobe of the $\mathrm{H}_{2} \mathrm{O}$ maser jet. Despite the different speeds (up to $\sim 100 \mathrm{~km} \mathrm{~s}^{-1}$ for $\mathrm{H}_{2} \mathrm{O}$ and only $\sim 5 \mathrm{~km} \mathrm{~s}^{-1}$ for $\mathrm{CH}_{3} \mathrm{OH}$ ), this suggests a relationship between the two. Second, the mean velocity of group 1 features along the line of sight is $-6.9 \mathrm{~km} \mathrm{~s}^{-1}$, which differs by $-3.4 \mathrm{~km} \mathrm{~s}^{-1}$ with respect to the systemic LSR velocity of $-3.5 \mathrm{~km} \mathrm{~s}^{-1}$. The mean distance of the same features from the nominal position of the star (starred symbol in Fig. 3) is 0 '.27 or $\sim 460$ AU. If the masers are undergoing Keplerian rotation about the $7 M_{\odot}$ star, the expected velocity is $\sim-3.7 \mathrm{~km} \mathrm{~s}^{-1}$, consistent with the value of $-3.4 \mathrm{~km} \mathrm{~s}^{-1}$ quoted above.

Based on this, we propose that group 1 masers trace the Keplerian disk (as proposed by Minier et al. 2001; and Edris et al. 2005). As for group 2 masers we speculate that they also lie in the disk, but very close to the transition region between the disk surface and the outer part of the jet. Although based on admittedly marginal evidence, this hypothesis may explain why the relative proper motions of these features (see Fig. 3) are roughly perpendicular to the plane of the disk. Besides, we will show in the following that also the line-of-sight velocities of group 2 masers (whose mean value is $-5.9 \mathrm{~km} \mathrm{~s}^{-1}$ ) differ from those of 


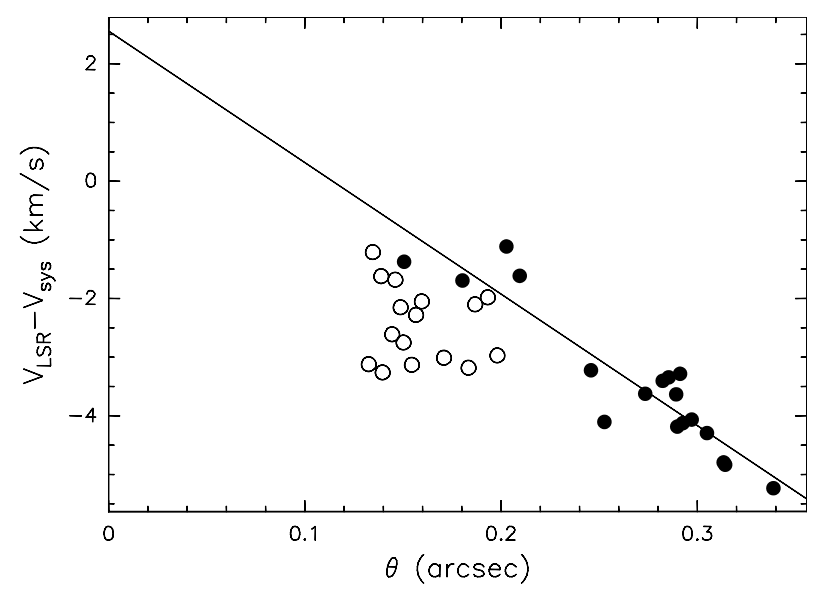

Fig. 4. Plot of the line-of-sight velocity of the $\mathrm{CH}_{3} \mathrm{OH}$ maser features, relative to the systemic LSR velocity $\left(-3.5 \mathrm{~km} \mathrm{~s}^{-1}\right)$, versus the angular separation from the nominal position of the star, measured along the dashed line in Fig. 3. Solid and empty circles denote, respectively, group 1 and group 2 features. The line is a linear fit to the points of group 1.

group 1 masers lending support to our speculation. Finally, we note that the number of maser spots without measurable proper motions is larger in group $2(40 \%)$ then in group $1(22 \%)$. This suggests that masers at the jet interface might be more persistent than those in the disk plane, consistent with the idea that radiative excitation of methanol masers may be more effective close to the surface than inside the densest parts of the disk.

\subsection{1. $\mathrm{CH}_{3} \mathrm{OH}$ maser group 1: the disk}

From Fig. 3 one can see that the nominal position of the star (obtained from the model fit to the $\mathrm{H}_{2} \mathrm{O}$ maser jet) lies right along the dashed line obtained from a linear fit to the features of group 1 . This is consistent with our hypothesis that these masers lie in the plane of the disk. One can thus study how the component of the rotation velocity along the line of sight depends on the projected distance from the star. This is shown in Fig. 4, where we plot the difference between the $\mathrm{CH}_{3} \mathrm{OH}$ maser velocity and the systemic velocity of IRAS $20126+4104\left(\sim-3.5 \mathrm{~km} \mathrm{~s}^{-1}\right)$ versus the angular distance, $\theta$, from the nominal position of the star, measured along the dashed line in Fig. 3. For the sake of comparison, we plot also the velocities of group 2 features.

Interestingly, for group 1 the velocity presents a linear dependence on $\theta$, different from a Keplerian disk where the rotation velocity should vary with the distance $R$ from the star as $R^{-1 / 2}$. However, if the masers are all lying in the disk at the same distance from the star, one can demonstrate that the line-of-sight component of the velocity is proportional to the projected distance from the star, as in Fig. 4. Why should the masing gas be confined to a narrow ring? One may speculate that the relevant physical parameters attain the values needed to trigger the $\mathrm{CH}_{3} \mathrm{OH}$ population inversion at a specific radius in the disk, because high densities near the star likely would quench the masers and low densities far from the star may provide too little gain for strong amplification.

In this model, the velocity along the line of sight should be zero at the position of the star, namely at $\theta=0$ in Fig. 4 . A linear fit to the solid circles in the figure gives $\left(V_{\mathrm{LSR}}-V_{\mathrm{sys}}\right)\left(\mathrm{km} \mathrm{s}^{-1}\right)=$ $(-22.4 \pm 1.1) \theta(\operatorname{arcsec})+(2.6 \pm 0.3)$, implying that $\left(V_{\mathrm{LSR}}-V_{\text {sys }}\right)=$ $0 \mathrm{~km} \mathrm{~s}^{-1}$ is attained for $\theta=0$.'11. Although different from zero, this offset is within the uncertainties on the nominal position of the star (obtained from our model fit to the $\mathrm{H}_{2} \mathrm{O}$ maser jet).

From the fit in Fig. 4 one can also compute a lower limit to the mass of the star. This is obtained assuming that the rotation speed of the farthest feature from the star is directed along the line of sight. Centrifugal equilibrium with $\sim 5 \mathrm{~km} \mathrm{~s}^{-1}$ at a radius of $0{ }^{\prime} 34-0{ }^{\prime} 11=0 . ' 23$ (or $\sim 390$ AU) implies a mass of $11 M_{\odot}$, consistent with the estimate of $7 \pm 3 M_{\odot}$ by Cesaroni et al. (2005).

We conclude that the group $1 \mathrm{CH}_{3} \mathrm{OH}$ maser features might be tracing a narrow ring inside the circumstellar disk in IRAS 20126+4104. A similar result has been obtained by Torstensson et al. (2008, 2010), who find that the $\mathrm{CH}_{3} \mathrm{OH}$ maser spots in Cep A appear to be all equidistant from the $7 \mathrm{~mm}$ continuum peak. This as well as other similar studies (Bartkiewicz et al. 2005, 2009; Vlemmings et al. 2010) seem to suggest that not only $\mathrm{CH}_{3} \mathrm{OH}$ masers form in disks, but also that they are excited at a specific radius rather than being randomly distributed all over the disk.

\subsection{2. $\mathrm{CH}_{3} \mathrm{OH}$ maser group 2: the jet}

In Fig. 4 one can see that all group 2 points fall below the trend described by group 1 points: as noted above, this appears to confirm a difference between the origins of the two maser groups. In particular, we suggest that the group 2 velocities might be affected by (blue-shifted) expansion along the jet, which would be entraining part of the material of the disk. In other words, we speculate that group 2 features could be tracing the outer regions of the jet, where the material is lifted from the disk surface and accelerated along the rotation axis. This scenario is reminiscent of MHD "disk winds" (see, e.g., Pesenti et al. 2004, and references therein), invoked to explain the formation of jets in YSOs. These differ from the alternative model of " $\mathrm{X}$ winds" (Shu et al. 2000) basically for two characteristics: the size of the region from which the wind originates and its speed. While $\mathrm{X}$ winds predict that the jet should form inside a radius of $\sim 0.02$ AU and reach speeds as large as a few $100 \mathrm{~km} \mathrm{~s}^{-1}$, disk winds should arise from a region of $\sim 1 \mathrm{AU}$ and be much less biased towards high speeds.

Under the working hypothesis that our interpretation is correct, one may wonder whether our findings could be used to discriminate between the two models: disk-wind and X-wind. In IRAS 20126+4104, the group $2 \mathrm{CH}_{3} \mathrm{OH}$ masers are moving at a speed of $\sim 5 \mathrm{~km} \mathrm{~s}^{-1}$ and are located at a radius of $\sim 300 \mathrm{AU}$ from the disk axis. While these values appear quite inconsistent with $\mathrm{X}$ winds, also a disk-wind interpretation is doubtful. In fact, $300 \mathrm{AU}$ is by far greater than the radius expected for the formation of a jet in this type of model and one should invoke a scaled-up version of disk winds to explain such a large value. However, disk wind models cannot be simply extrapolated to the high-mass case, because massive protostars reach the zero-age main sequence still undergoing heavy accretion, whose impact on jet formation is certainly non negligible. In addition, the role of magnetic fields relative to gravitational forces may be different in high-mass star formation with respect to low-mass.

While no safe conclusion can be drawn on the basis of the present data and even the jet interpretation for group 2 features is questionable, we believe that further proper motion studies of class II $\mathrm{CH}_{3} \mathrm{OH}$ masers in this source are a unique tool to investigate the structure and velocity field of the "twilight zone" between the disk and the jet, on spatial scales which cannot be investigated even with ALMA. 


\section{Summary and conclusions}

We performed multi-epoch water and $6.7 \mathrm{GHz}$ methanol maser VLBI observations towards the well known high-mass protostar IRAS 20126+4104. The $\mathrm{H}_{2} \mathrm{O}$ maser data are used to measure the annual parallax of the source and thus obtain an accurate distance of $1.64 \pm 0.05 \mathrm{kpc}$. This is consistent with the value adopted so far in the literature $(1.7 \mathrm{kpc})$ and confirms that IRAS $20126+4104$ is the best example of an early B-type protostar associated with a Keplerian disk and bipolar jet.

We also derived the absolute proper motions of the $\mathrm{CH}_{3} \mathrm{OH}$ masers, which gives the $3 \mathrm{D}$ velocity of the disk+star system. This can be used to correct the $\mathrm{H}_{2} \mathrm{O}$ maser velocities given by Moscadelli et al. (2005) and optimize their model fit. The bestfit parameters are qualitatively consistent with those obtained by Moscadelli et al. (2005), confirming that the jet lies very close to the plane of the sky and is roughly perpendicular to the disk.

By referring the proper motions of the $\mathrm{CH}_{3} \mathrm{OH}$ masers to a feature lying in the disk plane and analysing their distribution and kinematics, we identify two groups of maser features: one associated with the disk, the other possibly tracing the disk at the interface with the bipolar jet. We speculate that class $\mathrm{II} \mathrm{CH}_{3} \mathrm{OH}$ masers are an ideal tracer to investigate the dense molecular gas entrained at the basis of the jet.

Acknowledgements. We thank Francesca Bacciotti for useful discussions about the properties of $\mathrm{X}$-winds and disk-winds. We also thank the referee, Gary Fuller, for his careful reading of the manuscript and useful suggestions. This work has benefited from research funding from the European Community's sixth Framework Programme under RadioNet R113CT 20035058187 and has been partly supported by the European Community Framework Programme 7, Advanced Radio Astronomy in Europe, grant agreement No. 227290.

\section{References}

Bartkiewicz, A., Szymczak, M., \& van Langevelde, H. J. 2005, A\&A, 442, L61 Bartkiewicz, A., Szymczak, M., van Langevelde, H. J., Richards, A. M. S., \& Pihlström, Y. M. 2009, A\&A, 502, 155

Caratti o Garatti, A., Froebrich, D., Eislöffel, J., Giannini, T., \& Nisini, B. 2008, A\&A, 485, 137
Cesaroni, R., Felli, M., Testi, L., Walmsley, C. M., \& Olmi, L. 1997, A\&A, 325, 725

Cesaroni, R., Felli, M., Jenness, T., et al. 1999, A\&A, 345, 949

Cesaroni, R., Neri, R., Olmi, L., et al. 2005, A\&A, 434, 1039

Cesaroni, R., Galli, D., Lodato, G., Walmsley, M., \& Zhang, Q. 2006, Nature, 444, 703

Cesaroni, R., Galli, D., Lodato, G., Walmsley, C. M., \& Zhang, Q. 2007, in Protostars and Planets V, ed. B. Reipurth, D. Jewitt, \& K. Keil (Tucson: Univ. of Arizona Press), 197

De Buizer, J. M. 2007, ApJ, 654, L147

Edris, K. A., Fuller, G. A., Cohen, R. J., \& Etoka, S. 2005, A\&A, 434, 213

Felli, M., Brand, J., Cesaroni, R., et al. 2007, A\&A, 476, 373

Hofner, P., Cesaroni, R., Rodríguez, L. F., \& Martí, J. 1999, A\&A, 345, L43

Hofner, P., Cesaroni, R., Olmi, L., et al. 2007, A\&A, 465, 197

Krumholz, M. R., Klein, R. I., McKee, C. F., Offner, S. S. R., \& Cunningham, A. J. 2009, Science, 323, 754

Lebrón, M., Beuther, H., Schilke, P., \& Stanke, Th. 2006, A\&A, 448, 1037

Mihalas, D., \& Binney, J. 1981, Galactic Astronomy (San Francisco: W.H. Freeman and Company)

Minier, V., Booth, R. S., \& Conway, J. E. 2000, A\&A, 362, 1093

Minier, V., Conway, J. E., \& Booth, R. S. 2001, A\&A, 369, 278

Moscadelli, L., Cesaroni, R., \& Rioja, M. J. 2000, A\&A, 360, 663

Moscadelli, L., Cesaroni, R., \& Rioja, M. J. 2005, A\&A, 438, 889

Norris, R. P., Byleveld, S. E., Diamond, P. J., et al. 1998, ApJ, 508, 275

Palla, F., \& Stahler, S. W. 1993, ApJ, 418, 414

Pesenti, N., Dougados, C., Cabrit, S., et al. 2004, A\&A, 416, L9

Pestalozzi, M. R., Elitzur, M., \& Conway, J. E. 2009, A\&A, 501, 999

Reid, M. J., Menten, K. M., Brunthaler, A., et al. 2009a, ApJ, 693, 397

Reid, M. J., Menten, K. M., Zheng, X. W., et al. 2009b, ApJ, 700, 137

Sanna, A., Moscadelli, L., Cesaroni, R., et al. 2010, A\&A, 517, A71

Shepherd, D. S., Yu, K. C., Bally, J., \& Testi, L. 2000, ApJ, 535, 833

Shinnaga, H., Phillips, T. G., Furuya, R. S., \& Cesaroni, R. 2008, ApJ, 682, 1103

Shu, F. H., Najita, J. R., Shang, H., \& Li, Z.-Y. 2000, in Protostars and Planets IV, ed. V. Mannings, A. Boss, \& S. Russel (Tucson: Univ. of Arizona Press), 789

Sridharan, T. K., Williams, S. J., \& Fuller, G. A. 2005, ApJ, 631, L73

Su, Y.-N., Liu, S.-Y., Chen, H.-R., Zhang, Q., \& Cesaroni, R. 2007, ApJ, 671, 571

Torstensson, K., van Langevelde, H. J., Vlemmings, W., \& van der Tak, F. 2008, 9th EVN Symposium, 39

Torstensson, K. J. E., van Langevelde, H. J., Vlemmings, W. H. T., \& Bourke, S. 2010, A\&A, 526, A38

Trinidad, M. A., Curiel, S., Migenes, V., et al. 2005, AJ, 130, 2206

Vlemmings, W. H. T., Surcis, G., Torstensson, K. J. E., \& van Langevelde, H. J. 2010, MNRAS, 404, 134

Wilking, B. A., Blackwell, J. H., \& Mundy, L. G. 1990, AJ, 100, 758

Zhang, Q., Hunter, T. R., \& Sridharan, T. K. 1998, ApJ, 505, L151 